\title{
UNIVERSAL CRITICAL QUANTUM PROPERTIES OF CUPRATE SUPERCONDUCTORS
}

\author{
T. SCHNEIDER \\ IBM Research Division, Zurich Research Laboratory \\ Säumerstr. 4, 8803 Rüschlikon, Switzerland \\ and \\ Physik-Institut, University of Zürich, 8057 Zürich, Switzerland
}

\begin{abstract}
Using the scaling theory of quantum critical phenomena we explore the occurrence of universal critical behavior at the insulator-to-superconductor and superconductor-to-normal state transitions at zero temperature. Experimentally, these phase transitions are driven by doping and correspond to critical end points of the phase transition line in the temperature-hole concentration plane. Provided that the order parameter is a complex scalar in two dimensions, and that the London relation between superfluid number density and magnetic penetration depth holds, the scaling theory predicts universal behavior close to the insulator-to-superconductor transition. In particular, transition temperature and zero temperature penetration depth are universally related and the sheet resistance adopts a universal value. These predictions agree remarkably well with available experimental data and provide useful constraints for a microscopic theory.
\end{abstract}

PACS numbers: 74.72.-h, 74.25.-q, 74.62.-c, 74.62.Dh

Many physical properties of cuprate superconductors depend on hole doping. The generic doping dependence of the transition temperature $T_{\mathrm{c}}$ is depicted in Fig. 1. At a certain doping level, the so-called underdoped limit, these materials undergo at the hole concentration $x_{\mathrm{u}}$ and $T>0$ a transition from insulator to anomalous metal, and at $T=0$ a transition from insulator to superconductor. As $x$ is increased, $T_{\mathrm{c}}$ rises and reaches its maximum at $x_{\mathrm{m}}$. This behavior is shared by many cuprates, including $\mathrm{YBa}_{2} \mathrm{Cu}_{3} \mathrm{O}_{7-\delta}$ [1], $\mathrm{La}_{2-x} \mathrm{Sr}_{x} \mathrm{CuO}_{4-\delta}$ [2], $\mathrm{Y}_{2} \mathrm{Ba}_{4} \mathrm{Cu}_{7} \mathrm{O}_{15+\delta}$ [3], $\mathrm{Y}_{1-x} \mathrm{Pr}_{x} \mathrm{Ba}_{2} \mathrm{Cu}_{3} \mathrm{O}_{6.97}$ [4], $\mathrm{Tl}_{2} \mathrm{Ba}_{2} \mathrm{CuO}_{6+\delta}$ [5], $\mathrm{Bi}_{2} \mathrm{Sr}_{2} \mathrm{CuO}_{6+\delta}$, $\mathrm{Bi}_{2} \mathrm{Sr}_{2} \mathrm{CaCu}_{2} \mathrm{O}_{8+\delta}$ [6], and $\mathrm{HgBa}_{2} \mathrm{CuO}_{4+\delta}$ [7]. In some compounds a further increase in the doping level leads to more metallic normal-state properties $[8,9]$, but $T_{\mathrm{c}}$ decreases and vanishes in the overdoped limit $x_{\mathrm{o}}$. Here, these materials undergo at $T=0$ a superconductor-to-normal-state transition. A prominent example is $\mathrm{La}_{2-x} \mathrm{Sr}_{x} \mathrm{CuO}_{4-\delta}$ [2].

Another essential and doping-dependent property is the effective mass anisotropy, characterized by $\gamma=\sqrt{M_{\perp} / M_{\|}}$. In optimally doped $\left(x=x_{\mathrm{m}}\right)$ $\mathrm{YBa}_{2} \mathrm{Cu}_{3} \mathrm{O}_{6.97}$ [10], $\mathrm{La}_{2-x} \mathrm{Sr}_{x} \mathrm{CuO}_{4}$ [11,12], and $\mathrm{Bi}_{2} \mathrm{Sr}_{2} \mathrm{CaCu}_{2} \mathrm{O}_{8}$ [13], $\gamma$ adopts 


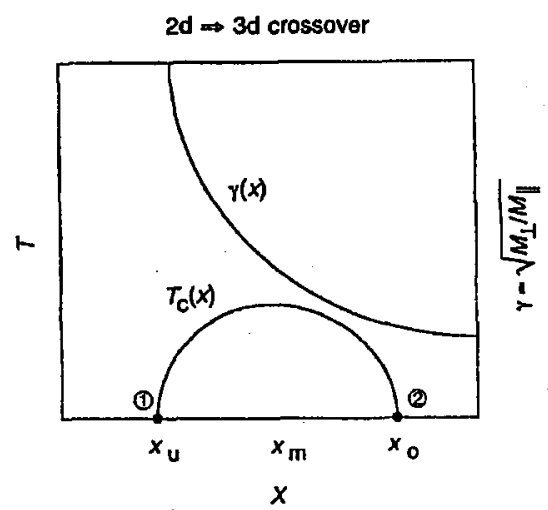

Fig. 1. Schematic phase diagram of cuprate superconductors. At $T=0$ and with increasing hole concentration $x$ the materials undergo a $2 d$ to $3 d$ crossover, at $x_{\mathrm{u}}$ an insulator-superconductor and at $x_{0}$ a superconductor-to-normal state transition. End point 1 denotes the $2 d$ quantum-critical point of the insulator-to-superconductor transition and 2 - the anisotropic $3 d$ quantum-critical point of the superconductor-to-normal state transition. 1 and 2 are the end points of the phase transition line $T_{\mathrm{c}}(x)$. $\gamma=\sqrt{M_{\perp} / M_{\|}}$is the effective mass anisotropy.

the values 5, 20, and > 150, respectively. Moreover, in both $\mathrm{La}_{2-x} \mathrm{Sr}_{x} \mathrm{CuO}_{4}$ [12] and $\mathrm{YBa}_{2} \mathrm{Cu}_{3} \mathrm{O}_{6.97}[10], \gamma$ becomes very large by approaching the underdoped limit. Noting then that $\gamma \rightarrow \infty$ represents the two-dimensional (2d) limit, it becomes evident that $2 d$ fluctuations dominate in the underdoped limit, whereas with increasing doping level a $2 d-3 d$ crossover occurs. Nevertheless, for sufficiently large $\gamma$ values, the regime where $3 d$ fluctuations dominate might be experimentally inaccessible.

As there is a phase transition line with two end points at $T=0$ ( 1 and 2 ), a $2 d$ to $3 d$ crossover, and strong evidence of the importance of critical fluctuations at finite temperature $[14,15]$, it seems natural to explore the problem of a consistent description of the doping dependence of various superconducting properties with the scaling theory of quantum critical phenomena [16]. Using this approach we show that at the critical end point 1 the relations

$$
T_{\mathrm{c}}(\delta)=\frac{\Phi_{0}^{2}}{Q_{0} 16 \pi^{3} k_{\mathrm{B}}} \frac{d_{\mathrm{s}}}{\lambda_{\|}^{2}(\delta, T=0)}, \quad \tilde{\rho}=\frac{\rho}{d_{\mathrm{s}}}=\frac{1}{\sigma_{0}} \frac{h}{4 e^{2}}=\frac{1}{\sigma_{0}} 6.5 \mathrm{k} \Omega
$$

are universal, provided that the order parameter is a complex scalar in $d=2$ and the London relation between superfluid number density and magnetic penetration depth holds. The parameter $\delta=x-x_{\mathrm{u}}$ measures the distance from the critical point $1, d_{\mathrm{s}}$ is the thickness of the $d=2$ superconducting unit, $\lambda_{\|}(T=0)$ - the magnetic penetration depth parallel to the layers, and $\rho$ - the sheet resistance. $Q_{0}$ and $\sigma_{0}$ are constants which adopt model-dependent universal values. The finite-temperature analog of the relation between $T_{\mathbf{c}}(\delta)$ and $\lambda_{\|}^{-2}(\delta, T=0)$ is $k_{\mathrm{B}} T_{\mathrm{KT}}=\left(\Phi_{0}^{2} / 8 \pi^{2}\right) d_{\mathrm{s}} \lambda_{\|}^{-2}\left(T_{\mathrm{KT}}\right)$, yielding the universal NelsonKosterlitz jump [17] for $\lambda_{\|}^{-2}(T)$ at the Kosterlitz-Thouless transition temperature 
$T_{\mathrm{KT}}$ in $d=2$. In Fig. 2 we depicted $\mu \mathrm{SR}$ data and magnetic measurements in terms of $T_{\mathrm{c}}$ versus $1 / \lambda_{\|}^{2}(T \rightarrow 0)$ of $\mathrm{La}_{2-x} \mathrm{Sr}_{x} \mathrm{CuO}_{4}[18,19], \mathrm{YBa}_{2} \mathrm{Cu}_{3} \mathrm{O}_{7-\delta}$ [1], and $\mathrm{Y}_{1-x} \mathrm{Pr}_{x} \mathrm{Ba}_{2} \mathrm{Cu}_{3} \mathrm{O}_{6.97}$ [4]. As $T_{\mathrm{c}}$ approaches the critical end point 1 (Fig. 1), the data merges into the single line

$$
T_{\mathrm{c}} \approx 3.57 \times 10^{8} \frac{1}{\lambda_{\|}^{2}(T=0)}
$$

with $T_{\mathrm{c}}$ in units of $\mathrm{K}$ and $\lambda_{\|}(0)$ in $\AA$. Thus, as far as superconductivity is concerned, the bulk behaves in the limit $x \rightarrow x_{\mathrm{u}}$ like a stack of uncoupled $2 d$ units of thickness $d_{\mathrm{s}} \approx \mathrm{Q}_{0} \times 5.7 \AA$.

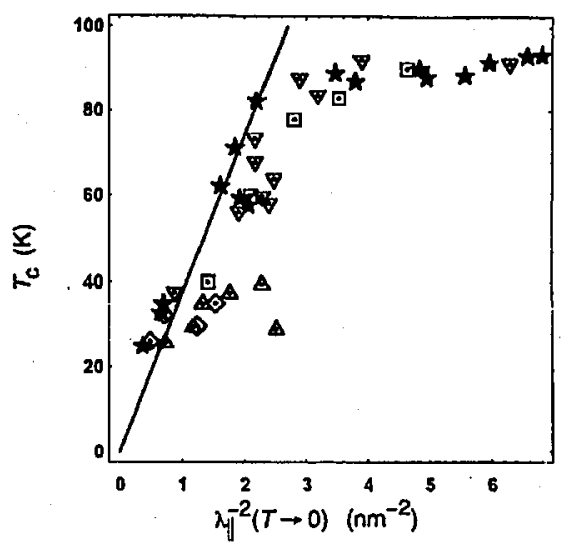

Fig. 2. $T_{\mathrm{c}}$ versus $\lambda_{\|}^{-2}(T \rightarrow 0)$ as obtained from $\mu \mathrm{SR}$ and magnetic measurements: $\mathrm{La}_{2-x} \mathrm{Sr}_{x} \mathrm{CuO}_{4}: \diamond, \mathrm{mag}, \Delta, \mu \mathrm{SR}[14,18,19] ; \mathrm{YBa}_{2} \mathrm{Cu}_{3} \mathrm{O}_{7-\delta}: \nabla, \operatorname{mag}, *, \mu \mathrm{SR}[1,14]$; $\mathrm{Y}_{1-x} \mathrm{Pr}_{x} \mathrm{Ba}_{2} \mathrm{Cu}_{3} \mathrm{O}_{6.97}:$ 口, $\mu \mathrm{SR}[4]$.

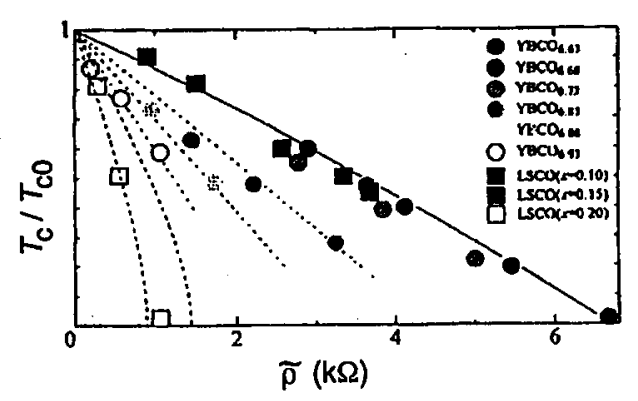

Fig. 3. Normalized critical temperature versus in-plane $2 d$ residual resistance taken from Ref. [20]. The solid curve is a line at which all the data in the underdoped regime merge. $T_{\mathrm{co}}$ denotes the transition temperature of the $\mathrm{Zn}$-free compound.

There is also considerable experimental evidence that the sheet resistance tends to a universal value at the insulator-to-superconductor transition 1. Fig- 
ure 3 shows $T_{\mathrm{c}}$ plotted versus the in-plane $2 d$ resistance per $\mathrm{CuO}_{2}$ plane for Zn-substituted $\mathrm{YBa}_{2} \mathrm{Cu}_{3} \mathrm{O}_{7-\delta}$ and $\mathrm{La}_{2-x} \mathrm{Sr}_{x} \mathrm{CuO}_{4}$ [20]. For the underdoped materials the data collapses onto the solid line, which tends in the limit $T_{\mathfrak{c}} \rightarrow 0$ to $6.5 \mathrm{k} \Omega$. Thus, at the insulator-to-superconductor transition 1 the underdoped compounds exhibit, in agreement with Eq. (1), true metallic and universal resistance. In contrast, the higher-doped cuprates show quite distinct $T_{\mathrm{c}}$ versus $\rho$ curves, which are strongly dependent on the doped-hole concentration. Closely related behavior has been found in $\mathrm{YBa}_{2} \mathrm{Cu}_{3} \mathrm{O}_{7-\delta}$ as the film was thinned down by ion bombardment [21] and in ultrathin $\mathrm{DyBa}_{2} \mathrm{Cu}_{3} \mathrm{O}_{7-\delta}$ films [22]. Superconductivity was found to disappear roughly at the sheet resistance $6.5 \mathrm{k} \Omega$.

We have seen that the predictions of the scaling theory of quantum critical phenomena in $d=2$ (Eq. (1)) agree remarkably well with a vailable experimental data. Noting that this formalism, supplemented by the critical properties of end points 1 and 2, also describes the asymptotic doping dependence, one expects this approach to provide a coherent description of the doping dependence of the superconducting properties. To substantiate this conjecture we begin, following Ref. [16], with a short sketch of the scaling theory of quantum critical phenomena. This also serves to derive Eq. (1) and relations used later on.

At $T=0$ a phase transition is driven by quantum fluctuations through parameters such as the chemical potential. Using this control parameter, the distance from the critical point is measured in terms of $\delta$. At $T=0$ and small $\delta$, one defines two correlation lengths, via the rate of decay of the Matsubara-Green function: the usual spatial correlation length in the disordered phase

$$
\xi=\xi_{0}^{+}|\delta|^{-\bar{\nu}}
$$

and the temporal correlation length

$$
\xi_{\tau}=\xi_{\tau 0}^{ \pm}|\delta|^{-\nu_{\tau}}
$$

The dynamic critical exponent $z$ is defined by the ratio

$$
z=\frac{\nu_{\tau}}{\bar{\nu}}
$$

Indeed, the inherent quantum dynamics determines the temporal evolution, which is generally different from the spatial evolution. The characteristic frequency scale $\Omega$ is determined from the dynamics as $\Omega \propto|\xi|^{-z} \propto|\delta|^{-z \bar{\nu}}$. Analogous to the classical hyperscaling expression for the free-energy density, one requires that the singular part of the free-energy density $f_{\mathrm{s}}$ in the correlation volume, $f_{\mathrm{s}} \prod_{i=1}^{d} \xi_{i} \xi_{\tau}$, is universal when $|\delta| \rightarrow 0$. The parameter $\xi_{i}$ denotes the spatial correlation length in direction $(i=(\|, \perp))$. Accordingly

$$
f_{\mathrm{s}} \propto \prod_{i=1}^{d} \xi_{i}^{-1} \xi_{\tau}^{-1} \propto|\delta|^{2-\bar{a}}=|\delta|^{\bar{\nu}(d+z)} .
$$

This yields the generalized hyperscaling relation

$$
2-\bar{\alpha}=\bar{\nu}(d+z) \text {. }
$$

Note that $\bar{\alpha}$ is defined via $-\partial^{2} f_{\mathrm{s}} / \partial \delta^{2}=\left(A_{ \pm} / \bar{\alpha}\right)|\delta|^{-\bar{\alpha}}$. The occurrence of superfluidity is conveniently described in terms of the free energy density in the presence of an imposed order-parameter twist with wave vector $k_{i}$. The extension of Eq. (6) is

$$
f_{\mathrm{s}} \propto|\delta|^{2-\vec{\alpha}} \Phi^{-}\left(C_{i} k_{i}|\delta|^{-\vec{\nu}}, \ldots\right)
$$


from which one derives for the helicity modulus

$$
Y_{i}=\left.\frac{\partial^{2} f_{s}}{\partial k_{i}^{2}}\right|_{k_{i}=0} \propto|\delta|^{2-\bar{\alpha}-2 \bar{\nu}} .
$$

The related transverse correlation length is then defined as

$$
\xi_{i}^{\mathrm{T}}=\left(Y_{i} \xi_{\tau}\right)^{1 /(2-d)}=\xi_{i 0}^{\mathrm{T}} \delta^{-\bar{\nu}} .
$$

Equation (8) can be extended to finite temperatures and finite frequency. The temperature and frequency-dependent helicity modulus is then defined in terms of the temporal Fourier transform of the current-current correlation function. The resulting scaling form is

$$
Y_{i}(\delta, T, \omega) \propto|\delta|^{2-\bar{\alpha}-2 \bar{\nu}} Y_{i}\left(C_{i} \hbar \omega|\delta|^{-z \bar{\nu}}, D_{i} \beta^{-1}|\delta|^{-z \bar{\nu}}\right) .
$$

We now assume that there is a line of finite temperature phase transitions $T_{\mathrm{c}}(\delta)$ ending at $T=0$ and $\delta=0$. The scaling form (Eq. (11)) taken at $\omega=0$ then reveals that

$$
k_{\mathrm{B}} T_{\mathrm{c}} \propto D|\delta|^{z \bar{\nu}} \text {. }
$$

Moreover, combining Eqs. (11) and (12), it follows that

$$
\lim _{\delta \rightarrow 0} \frac{Y_{i}(\delta)}{k_{\mathrm{B}} T_{\mathrm{c}}(\delta)} \propto|\delta|^{2-\bar{\alpha}-2 \bar{\nu}-z \bar{\nu}} \propto \xi_{i}^{2-d}
$$

is a universal number in $d=2$, namely

$$
\lim _{\delta \rightarrow 0} \frac{Y_{i}(\delta)}{k_{\mathrm{B}} T_{\mathrm{c}}(\delta)}=Q_{0}
$$

Clearly, $Q_{0}$ will adopt a characteristic value within a universality class. Using the relation between the helicity modulus and the superfluid aerial density in $d=2$ (pairs per unit area), as well as the relation between aerial superfluid density and bulk penetration depth the helicity modulus reduces to

$$
Y_{\|}(\delta, T=0)=\frac{\hbar^{2} \tilde{n}_{\mathbf{s}}}{M_{\|}}=\frac{\Phi_{0}^{2}}{16 \pi^{3}} \frac{d_{\mathbf{s}}}{\lambda_{\|}^{2}(\delta, T=0)},
$$

where $d_{\mathrm{s}}$ is the thickness of the superconducting units in $d=2$. Combining then Eqs. (14) and (15) we recover the universal relation given in Eq. (1). For $d \neq 2$, however, Eqs. (12) and (13) lead to

$$
Y_{i}(\delta, T=0) \propto T_{\mathrm{c}}(\delta)^{(\delta+z-2) / z} \propto \frac{\Phi_{0}^{2}}{16 \pi^{3}} \frac{1}{\lambda_{\|}^{2}(\delta, T=0)}
$$

with a nonuniversal coefficient of proportionality. It is important to emphasize that relation (14) holds generally for a system where the order parameter is a complex scalar, whereas relations (1) and (16) require that the London relation between the superfluid number density and the magnetic penetration depth holds.

The frequency-dependent conductivity is given by

$$
\sigma(\delta, T, \omega)=\frac{4 e^{2}}{h} \frac{Y(\delta, T,-\mathrm{i} \omega)}{-\mathrm{i} \hbar \omega},
$$

where $2 e$ is the charge of the pair. Using Eq. (11) and approaching the critical point at $T=0, \omega=0$, and $\delta=0$ along a path in such a way that $C_{i} \hbar \omega|\delta|^{-z \bar{\nu}}=$ 
$x, y=D_{i} \beta^{-1}|\delta|^{-z \bar{\nu}}$ approach some fixed value $x_{0}$ and $y_{0}$, one finds with the aid of the hyperscaling relation (7)

$$
\sigma_{\text {sing }}=\frac{4 e^{2}}{h}(\xi)^{-(d-2)} \sigma_{0}
$$

and the resistance should satisfy the scaling relation

$$
\rho(\delta, T)=(\xi)^{d-2} \frac{h}{4 e^{2}} Q\left(\frac{b \delta}{T^{1 / z v}}\right)
$$

yielding for $d=2$ the limiting universal resistivity given by Eq. (1).

We have seen that the scaling theory yields useful relations between critical exponents and discloses universal relations between critical amplitudes. It does not provide, however, the values of the critical exponents and the universal constants. This is because the universal scaling functions, the critical exponent relation, etc. are satisfied regardless of the particular universality class. A characteristic feature of the universality class are the critical exponents. Assuming that the distance from the critical point 1 is measured by $\delta=x-x_{\mathrm{u}}$ we obtain from Eqs. (7), (12), (13) and (15) in $d=2$ for the asymptotic behavior

$$
\lambda_{\|}^{2}(T=0)=\lambda_{\| \mathrm{u}}^{2}(T=0)\left(x-x_{\mathrm{u}}\right)^{-z \bar{\nu}}, \quad T_{\mathrm{c}}=r_{\mathrm{u}}\left(x-x_{\mathrm{u}}\right)^{z \bar{\nu}} .
$$

Equations (14) and (15) require that the critical amplitude combination

$$
\frac{r_{\mathrm{u}} \lambda_{\| \mathrm{u}}^{2}}{d_{\mathrm{s}}}=\frac{\Phi_{0}^{2}}{16 \pi^{3} Q_{0}}
$$

be universal. The available experimental data for the doping dependence of $T_{\mathrm{c}}[2,20]$ and $\lambda_{\|}(T=0)[18,19]$ is shown in Figs. 4 and 5 for $\mathrm{La}_{2-x} \mathrm{Sr}_{x} \mathrm{CuO}_{4}$. It is too sparse, however, to estimate $z \bar{\nu}$, the critical amplitudes $\left(\lambda_{\| u}, r_{\mathrm{u}}\right)$, and the location of the critical point $\left(x_{\mathrm{u}}\right)$. For this reason we explore the consistency with the generic insulator-to-superconductor transition of preformed pairs, where $z \bar{\nu}=1, z=2$, and $\bar{\nu}=1 / 2$ in all dimensions [23]. For $x_{\mathrm{u}}=0.05$ the dashed lines in Figs. 4 and 5 correspond to the critical amplitudes $r_{\mathrm{u}} \approx 880 \mathrm{~K}$ and $\lambda_{\| \mathrm{u}}^{2} \approx 3.84 \times 10^{5} \AA^{2}$, yielding for the universal prefactor in Eq. (1) the estimate $3.38 \times 10^{8}$, which is close to the value derived from $\mu \mathrm{SR}$ data (Eq. (2)). To strengthen this analysis of the rather sparse experimental data, we note that the exponents $z \bar{\nu}=1, z=2$, and $\bar{\nu}=1 / 2$ describe the insulator-to-superconductor transition of preformed pairs. A characteristic feature of this transition is that at finite temperature, close to $x_{u}$ and below a mean-field transition temperature, the electrons form pairs, but the true transition temperature $T_{\mathrm{c}}^{\mathrm{MF}}$ is lowered by the enhanced phase fluctuations in $2 d$. Consequently, pairs are expected to occur for $T<T_{\mathrm{c}}^{\mathrm{MF}}$ and the effects of pairing should then manifest themselves as a suppression of low-energy excitations, that is, in terms of a gap. The existence of such a gap in underdoped cuprates, opening below $T_{\mathrm{c}}^{\mathrm{MF}}$ is well confirmed by a variety of measurements, including NMR [24], optical conductivity [25], and angular resolved photoemission [26].

To present our final application we speculate briefly about applying the scaling theory of quantum critical phenomena to the superconductor-to-normal-state transition 2 (Fig. 1). The experimental evidence of more isotropic and metallic normal-state properties in the overdoped regime $[8,9,12]$ indicates that in this doping regime the two aforementioned energy scales, the binding energy of the 


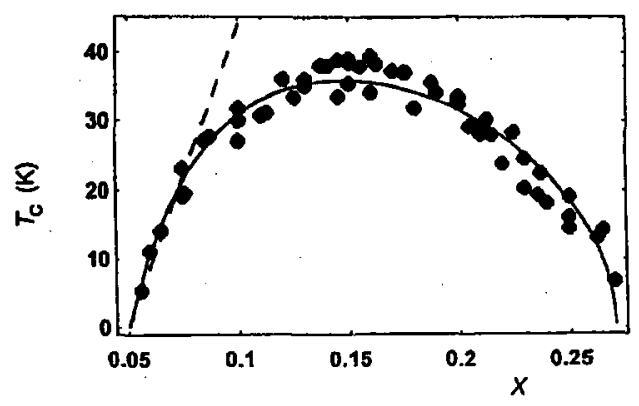

Fig. 4. $T_{c}$ versus $x$ for $\mathrm{La}_{2-x} \mathrm{Sr}_{x} \mathrm{CuO}_{4}$ taken from Refs. [2, 12, 20]. The dashed line is a fit to Eq. (20) yielding for the critical amplitude the estimate $r_{\mathrm{u}} \approx 880 \mathrm{~K}$. The solid curve is a guide to the eye.

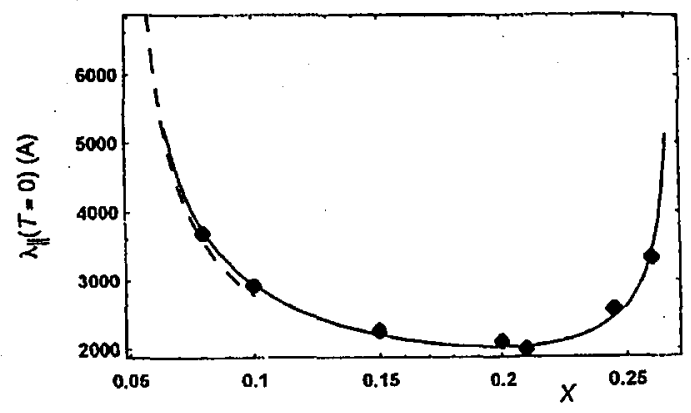

Fig. 5. $\lambda_{\|}(T=0)$ versus $x$ for $\mathrm{La}_{2-x} \mathrm{Sr}_{x} \mathrm{CuO}_{4}$ derived from $\mu \mathrm{SR}$ data of Refs. [18, 19]. The dashed line is a fit to $\mathrm{Eq}$. (20) yielding for the critical amplitude the estimate $\lambda_{\| 4}^{2} \approx 3.84 \times 10^{5} \AA^{2}$. The solid curve is a guide to the eye.

pairs and the condensation energy approach one another. For these reasons a BCS-Eliashberg type of superconductivity might be expected. It appears nonetheless likely that the asymptotic critical properties of transition 2 are insensitive to the fermionic degrees of freedom. Indeed, the critical points along the phase transition line $T_{\mathrm{c}}(x)$ and Bose condensation appear to belong to the same universality class $(3 d-x y)[14,15]$, the difference between the normal bosonic and fermionic phases notwithstanding. Thus, we expect that the critical properties along the phase transition line $T_{\mathrm{c}}(x)$ and at the $3 d$ critical end point can be properly described in terms of neutral bosons. Relation (16) then reduces to

$$
T_{\mathrm{c}}\left(x_{\mathrm{o}}-x\right) \propto\left[\frac{1}{\lambda_{\|}^{2}\left(x_{\mathrm{o}}-x, T=0\right)}\right]^{z /(1+z)}
$$

with a nonuniversal coefficient of proportionality. Moreover, analogous to Eq. (20), the asymptotic doping dependence of $T_{\mathrm{c}}$ and the zero temperature penetration 
depth $\lambda_{\|}(T=0)$ obey

$$
\lambda_{\|}^{2}(T=0)=\lambda_{\| 0}^{2}(T=0)\left(x_{\circ}-x\right)^{-z \bar{\nu}}, \quad T_{\mathrm{c}}=r_{\circ}\left(x_{\circ}-x\right)^{z \bar{\nu}} .
$$

Unfortunately, even for $\mathrm{La}_{2-x} \mathrm{Sr}_{x} \mathrm{CuO}_{4}$ (Figs. 4 and 5) the available experimental data is sparse, and reliable estimates for the location of the critical end point as well as for the associated exponents and amplitudes cannot be extracted. From Fig. 6, which shows $T_{\mathrm{c}}$ versus $\sigma(T=0) \propto \lambda_{\|}^{-2}(T=0)$ for $\mathrm{La}_{2-x} \mathrm{Sr}_{x} \mathrm{CuO}_{4-\delta}$, $\mathrm{HgBa}_{2} \mathrm{CuO}_{4+\delta}$, and $\mathrm{Tl}_{2} \mathrm{Ba}_{2} \mathrm{CuO}_{6+\delta}[27,28]$, where the overdoped regime is accessible, there is some evidence of the nonuniversal behavior predicted by Eq. (22). Indeed, by approaching the critical end point 1 , the data of $\mathrm{La}_{2-x} \mathrm{Sr}_{x} \mathrm{CuO}_{4-\delta}$ and $\mathrm{HgBa}_{2} \mathrm{CuO}_{4+\delta}$ merge on the line describing the limiting universal behavior close to the $d=2$ insulator-to-superconductor transition, whereas the data for overdoped materials indicates that the approach to end point 2 is nonuniversal.

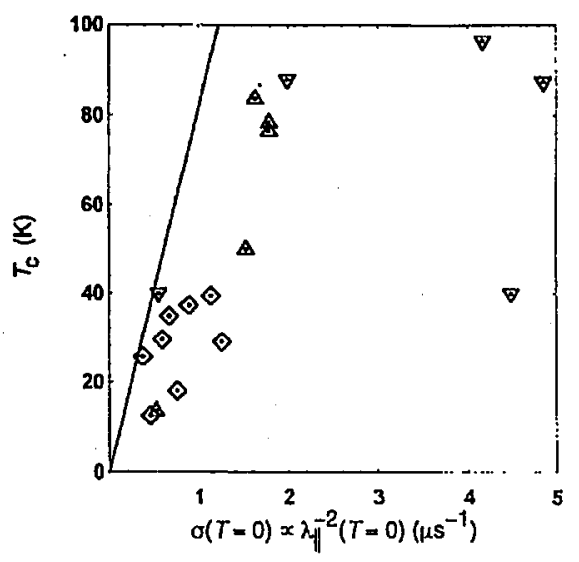

Fig. 6. $T_{\mathrm{c}}$ versus $\sigma(T=0) \propto \lambda_{\|}^{-2}(T=0)$ for $\mathrm{La}_{2-x} \mathrm{Sr}_{x} \mathrm{CuO}_{4-\delta}[18,19]$ : $\diamond, \mathrm{HgBa}_{2} \mathrm{CuO}_{4+\delta}[7]: \nabla$ and $\mathrm{Tl}_{2} \mathrm{Ba}_{2} \mathrm{CuO}_{6+\sigma}[27,28]: \Delta$. The solid line corresponds to the limiting universal behavior of Eq. (1) at the insulator-to-superconductor transition.

The considerations presented here are macroscopic and independent of the underlying pairing mechanism. However, some constraints on a microscopic theory emerge. (1) The order parameter of the superconducting state appears to be well described by a complex order parameter with an amplitude and phase. (2) The agreement between experimental data and the universal relations (1) clearly reveal that phase fluctuations are essential and predominant close to the insulator-to-superconductor transition 1. For this reason the BCS and Eliashberg theories, where the phase of the order parameter is unimportant for determining the value of $T_{\mathrm{c}}$, are not applicable. (3) The consistency with the bosonic insulator-to-superfluid transition at end point 1 to preformed pairs in the underdoped regime and pairing is then manifested in terms of a gap below $T_{\mathrm{c}}^{\mathrm{MF}}$ which is well confirmed by a variety of measurements. (4) As the doping level rises, the materials undergo a $2 d$ - to anisotropic $3 d$ crossover. (5) The experimental evidence for metallic normal-state 
properties in the overdoped regime requires that $T_{\mathrm{c}}$ and $T_{\mathrm{c}}^{\mathrm{MF}}$ approach one another. For these reasons phase fluctuations are no longer predominant and the BCS-Eliashberg mean-field theory might apply. In any case, close to the critical end point 2 the materials are anisotropic but $3 d$, which renders the relation between $T_{\mathrm{c}}$, and the zero temperature penetration depth nonuniversal.

I would like to thank many colleagues for discussions, particularly $\emptyset$. Fischer, Y. Jaccard, P. Martinoli, R. Micnas, M.II. Pedersen and J. Singer.

\section{References}

[1] P. Zimmermann, H. Keller, S.L. Lee, I.M. Savic, M. Warden, D. Zech, R. Cubitt, E.M. Forgan, E. Kaldis, J. Karpinski, C. Kruger, Phys. Rev. B 52, 541 (1995).

[2] J.B. Torrance, A. Bezinger, A.I. Nazzal, T.C. Huang, S.S.P. Parkin, D.T. Keane, S.J. LaPlaca, P.M. Horn, G.A. Ield, Phys. Rev. B 40, 8872 (1989).

[3] J.-Y. Genoud, T. Graf, G. Triscone, A. Junod, J. Muller, Physica C 192, 137 (1992).

[4] C.L. Seaman, J.J. Neumeier, M.B. Maple, L.P. Le, G.M., Luke, B.J. Sternlieb, Y.J. Uemura, J.H. Brewer, R. Kadano, R.F. Kiefel, S.R. Krietzman, T. Riseman, Phys. Rev. B 42, 6801 (1990).

[5] C. Opagiste, G. Triscone, M. Couach, T.K. Kondo, J.-L. Jorda, A. Junod, A.F. Khoder, J. Muller, Physica C 213, 17 (1993).

[6] W.A. Groen, D.M. de Leeuw, L.F. Feiner, Physica C 165, 55 (1990).

[7] C.W. Chu, Y. Cao, Q. Xiong, Y.Y. Xue, J. Superconduct. 8 , 393 (1995).

[8] Y. Iye, in Physical Properties of IIigh Temperature Superconductors III, Ed. D.M. Gimsberg, World Scientific, Singapore 1992, p. 285.

[9] B. Batlogg, H.Y. Hwang, H. Takagi, H.L. Kao, J. Kwo, R.J. Cava, J. Low. Temp. 95, 23 (1994).

[10] T.R. Chien, W.R. Datars, B.W. Veal, A.P. Paulikas, P. Kostic, Chun Gu, Y. Jiang, Physica C 229, 273 (1994).

[11] B. Janossy, D. Prost, S. Pekker, L. Fruchter, Physica C 181, 51 (1991).

[12] T. Kimura, K. Kishio, T. Kobayashi, Y. Nakayama, N. Motohira, K. Kitazawa, K. Yamafuji, Physica C 192, 247 (1992).

[13] Z.J. Huang, Y.Y. Xue, R.L. Meng, C.W. Chu, Phys. Rev. B 49, 4218 (1994).

[14] T. Schneider, H. Keller, Int'l J. Mod. Phys. B 8, 487 (1994).

[15] Y. Jaccard, T. Schneider, J.-P. Locquet, E.J. Williams, P. Martinoli, O. Fischer, Europhys. Lett. 34, 281 (1996).

[16] K. Kim, P.B. Weichman, Phys. Rev. B 43, 13583 (1991).

[17] D.R. Nelson, J.M. Kosterlitz, Phys. Rev. Lett. 39, 1201 (1977).

[18] Y.J. Uemura, in: Polarons and Bipolarons in High $T_{\mathrm{c}}$ Superconductors and Related Materials, Eds. E.K.H. Saljie, A.S. Alexandrov, W.Y. Liang, Cambridge University Press, Cambridge 1995, p. 453.

[19] J.G. Bednorz, private communication.

[20] Y. Fukuzumi, K. Mizuhashi, K. Takenaka, S. Uchida, Phys. Rev. Lett. 76, 684 (1996).

[21] A.F. Hebard, R.H. Eick, T. Siegrist, E. Coleman, Mater. Res. Soc. Symp. Proc. 169, 565 (1990).

[22] T. Wang, K.M. Beauchamp, D.D. Berkley, B.R. Johnson, J.-X. Liu, J. Zhang, A.M. Goldman, Phys. Rev. B 43, 8623 (1991). 
[23] M.P.A. Fisher, P.B. Weichman, G. Grinstein, D.S. Fisher, Phys. Rev. B 40, 546 (1989).

[24] M. Mehring, Appl. Mag. Reson. 3, 383 (1992).

[25] P. Wachter, B. Bucher, R. Pittini, Phys. Rev. B 49, 13164 (1994).

[26] H. Ding, T. Yokoya, J.C. Campuzano, T. Takahashi, M. Randeira, M.R. Norman, T. Mochiku, K. Kadowaki, J. Giapintzakis, Nature 382 (1996).

[27] Ch. Niedermayer, C. Bernhard, U. Binninger, H. Gluckler, J.L. Tallon, E.J. Ansaldo, J.I. Budnick, Phys. Rev. Lett. 71, 1764 (1993).

[28] Y.J. Uemura, A. Keren, L.P. Le, G.M. Luke, W.D. Wu, Y. Kubo, T. Manako, Y. Shimakawa, M. Subramanian, J.L. Cobb, J.T. Markert, Nature 364, 605 (1993). 